\title{
Editorial
}

\section{Planck 2018 results}

In this volume, we proudly present a special feature on the results from the ESA Planck mission, based on data released by ESA and the Planck Collaboration in July 2018. This 2018 data release has significantly lower systematic residuals for both Planck instruments, LFI and HFI, and a more accurate photometric calibration for HFI. These calibration improvements are most significant over the largest angular scales and for the polarized emission. The resulting frequency maps were used to separate the diffuse sky emission into maps of the cosmic microwave background, the thermal Sunyaev-Zeldovich effect, the cosmic infrared background, and the Galactic thermal dust, carbon monoxide, anomalous dust, free-free, and synchrotron emission.

The 12 articles in the special issue describe the released data products and present scientific results extracted by the Planck Collaboration from this data. The six-parameter $\Lambda \mathrm{CDM}$ model continues to provide an excellent fit to the cosmic microwave background data at high and low redshift. Planck measures five of those six parameters to better than $1 \%$, and together with external datasets, sets tight limits to many possible extensions of the model. Beyond those immediate results, the Planck 2018 dataset constitutes an essential treasure trove and will have lasting importance for both cosmology and foreground astrophysics.

We warmly thank Jan Tauber and the Planck Science Team for coordinating this special feature.

Joao Alves, Thierry Forveille, Laura Pentericci, Steve Shore Astronomy $\mathcal{E}$ Astrophysics Editors 